\title{
Thyroid dysfunction in very low birth weight preterm infants
}

\author{
Ji Hoon Lee, MD, Sung Woo Kim, MD, Ga Won Jeon, MD, PhD, Jong Beom Sin, MD, PhD \\ Department of Pediatrics, Inje University Busan Paik Hospital, Inje University College of Medicine, Busan, Korea
}

Purpose: Thyroid dysfunction is common in preterm infants. Congenital hypothyroidism causes neurodevelopmental impairment, which is preventable if properly treated. This study was conducted to describe the characteristics of thyroid dysfunction in very low birth weight infants (VLBWIS), evaluate risk factors of hypothyroidism, and suggest the reassessment of thyroid function with an initially normal thyroid-stimulating hormone (TSH) as part of a newborn screening test.

Methods: VLBWIs (January 2010 to December 2012) were divided into two groups according to dysfunction-specific thyroid hormone replacement therapy, and associated factors were evaluated.

Results: Of VLBWIs, 246 survivors were enrolled. Only 12.2\% (30/246) of enrolled subjects exhibited thyroid dysfunction requiring thyroid hormone replacement. Moreover, only one out of 30 subjects who required thyroid hormone treatment had abnormal thyroid function in the newborn screening test with measured TSH. Most of the subjects in the treatment group (22/30) exhibited delayed TSH elevation. Gestational age, Apgar score, antenatal steroids therapy, respiratory distress syndrome, patent ductus arteriosus, sepsis, intraventricular hemorrhage, postnatal steroids therapy, and duration of mechanical ventilation did not differ between the two groups. Birth weight was smaller and infants with small for gestational age were more frequent in the treatment group.

Conclusion: Physicians should not rule out suggested hypothyroidism, even when thyroid function of a newborn screening test is normal. We suggest retesting TSH and free thyroxine in high risk preterm infants with an initially normal TSH level using a newborn screening test.

Key words: Congenital hypothyroidism, Neonatal screening, Premature infant, Thyroid function tests, Very low birth weight infant

\section{Introduction}

Congenital hypothyroidism causes neurodevelopmental impairment which is preventable if treated properly ${ }^{11}$. Neurodevelopmental impairment caused by congenital hypothyroidism has decreased since the worldwide introduction of newborn screening tests.

Hypothyroidism is more common in preterm infants than in term infants, especially in sick preterm infants ${ }^{2}$. Preterm infants have lower levels of tri-iodothyronine (T3) and free thyroxine (T4) than term infants during the first several weeks, which is more severe in the smallest and least mature preterm infants ${ }^{3}$. Several physiologic and nonphysiologic factors are known to contribute to hypothyroidism in preterm infants, including an immaturity of the hypothalamic-pituitary-thyroid axis, an immaturity of thyroidal capacity to concentrate and synthesize iodine, an immaturity of thyroid hormonal metabolism, an increase of thyroid hormone requirement needs for thermogenesis and disease of preterm infants, iodine insufficiency and iodine excess ${ }^{33}$.

Transient hypothyroxinemia with decreased free T4 levels is self-limiting and common
Corresponding author: Ga Won Jeon, MD, PhD Department of Pediatrics, Inje University Busan Paik Hospital, Inje University College of Medicine, 75 Bokji-ro, Busanjin-gu, Busan 614-735, Korea Tel: +82-51-890-6497

Fax: +82-51-895-7785

E-mail: iamgawon@hanmail.net

Received: 1 July, 2014

Revised: 14 August, 2014

Accepted: 3 October, 2014
Copyright (C) 2015 by The Korean Pediatric Society

This is an open-access article distributed under the terms of the Creative Commons Attribution NonCommercial License (http://creativecommons.org/ licenses/by-nc/3.0/) which permits unrestricted noncommercial use, distribution, and reproduction in any medium, provided the original work is properly cited. 
in preterm infants. It is unclear whether the administration of thyroid hormone in transient hypothyroxinemia improves neurodevelopmental outcome, morbidity and mortality. Transient hyperthyrotropinemia with elevated thyroidstimulating hormone (TSH) is also common in preterm infants. Initial hyperthyrotropinemia can lead to persistent hyperthyrotropinemia or permanent hypothyroidism requiring thyroid hormone replacement therapy, or transient hypothyroidism that is self-limiting. Preterm infants are at significant risk of congenital hypothyroidism, which is unpredictable in its evolution to transient or permanent ${ }^{4)}$.

Three screening strategies are used for congenital hypothyroidism; a primary TSH method, a primary T4 method, and a combined primary TSH and T4 method. The newborn screening test in Korea uses a primary TSH method, which does not check free T4 levels. However, primary TSH is not elevated, which is particularly common in preterm infants, in thyroidbinding globulin (TBG) deficiency, central hypothyroidism, and hypothyroxinemia with delayed TSH elevation ${ }^{5}$. As a result, some congenital hypothyroidism cases with normal initial TSH levels go undetected, when using the single TSH approach 48 hours to 7 days postnatal.

Therefore, it is critical to screen congenital hypothyroidism in preterm infants, to prevent and minimize neurodevelopmental impairment. This study was conducted to describe the characteristics of thyroid dysfunction in very low birth weight preterm infants, evaluate risk factors of hypothyroidism in preterm infants, and recommend retesting a second or third specimen for thyroid function in high-risk preterm infants that registered normal initial TSH levels during the newborn screening test.

\section{Materials and methods}

\section{Patients}

Protocol of this study was reviewed and approved by the Institutional Review Board of the Inje University Busan Paik Hospital. Very low birth weight infants (VLBWIs) weighing less than $1,500 \mathrm{~g}$ admitted to the neonatal intensive care unit (NICU) of Inje University Busan Paik Hospital from January 2010 to December 2012 were enrolled.

Infants were allocated to a treatment group or nontreatment group based on whether thyroid hormone replacement therapy was administered for persistent thyroid dysfunction.

\section{Study protocol}

Clinical data were collected retrospectively from medical records. Various perinatal and postnatal factors that may influence thyroid dysfunction were evaluated.

Newborn screening test was done and thyroid function tests, including serum TSH and free T4 levels were measured at 7 days postnatal. If results were normal, levels were rechecked at 4 weeks postnatal and at discharge. If results were abnormal, levels were rechecked after 2 weeks. The patients with abnormal results in the 2nd exam were defined as 'persistent', and we started treatment. Thyroid hormone replacement therapy began previous to 4 weeks postnatal with 10 to $15 \mu \mathrm{g} / \mathrm{kg}$ of levothyroxine. Follow-up tests were done 2-4 weeks later after starting treatment. The treatment with levothyroxine was discontinued at 1-3 years postnatal. The patients with normalization in the 2nd exam were defined as 'transient' and didn't need treatment. Low free T4 was defined as a free T4 concentration $<0.7 \mathrm{mg} / \mathrm{dL}$, elevated TSH was defined as a TSH concentration $>20 \mathrm{mIU} / \mathrm{L}$, hypothyroidism was defined as a free $\mathrm{T} 4$ concentration $<0.7 \mathrm{mg} / \mathrm{dL}$ with a TSH concentration $>20 \mathrm{mIU} / \mathrm{L}^{5,6)}$.

Demographic factors comprised gestational age, birth weight, gender, Apgar score at 1 and 5 minutes, small for gestational age (SGA), antenatal magnesium sulfate therapy, antenatal corticosteroid therapy, maternal gestational diabetes mellitus (GDM), and maternal pregnancy induced hypertension (PIH).

Morbidity and outcome variables included respiratory distress syndrome (RDS), patent ductus arteriosus (PDA), sepsis, intraventricular hemorrhage (IVH), necrotizing enterocolitis (NEC), retinopathy of prematurity (ROP), postnatal steroids therapy, bronchopulmonary dysplasia (BPD), full enteral feeding day, duration of parenteral nutrition, duration of mechanical ventilation, and hospital stay. SGA was diagnosed when birth weight was below the 10th percentile on a Lubchenco growth curve ${ }^{77}$. Sepsis was limited to those with a positive blood culture showing clinical signs of systemic infection ${ }^{8}$. IVH was limited to a high grade ( $\geq$ grade III) $)^{9}$, NEC to a modified Bell stage $\geq \mathrm{II}^{10)}$, and ROP at a high stage requiring laser therapy ${ }^{11}$. BPD was defined as an oxygen dependency for the first 28 days of life and categorized as mild, moderate, and severe by the severity ${ }^{12)}$. The full enteral feeding day was defined as a postnatal day to achieve $120 \mathrm{~mL} /$ $\mathrm{kg} /$ day of feeding.

\section{Statistical analysis}

For continuous variables, a $t$ test or Mann-Whitney $U$ test was performed. For nominal variables, a chi-square test or Fisher exact test was performed. Statistical analysis was performed using SAS 3.0 (SAS Institute Inc., Cary, NC, USA). Data are given as mean \pm standard deviation. $P$ values of $<0.05$ were con $\neg$ sidered statistically significant.

\section{Results}

\section{Thyroid function test}

From January 2010 to December 2012, 246 VLBWIs who 


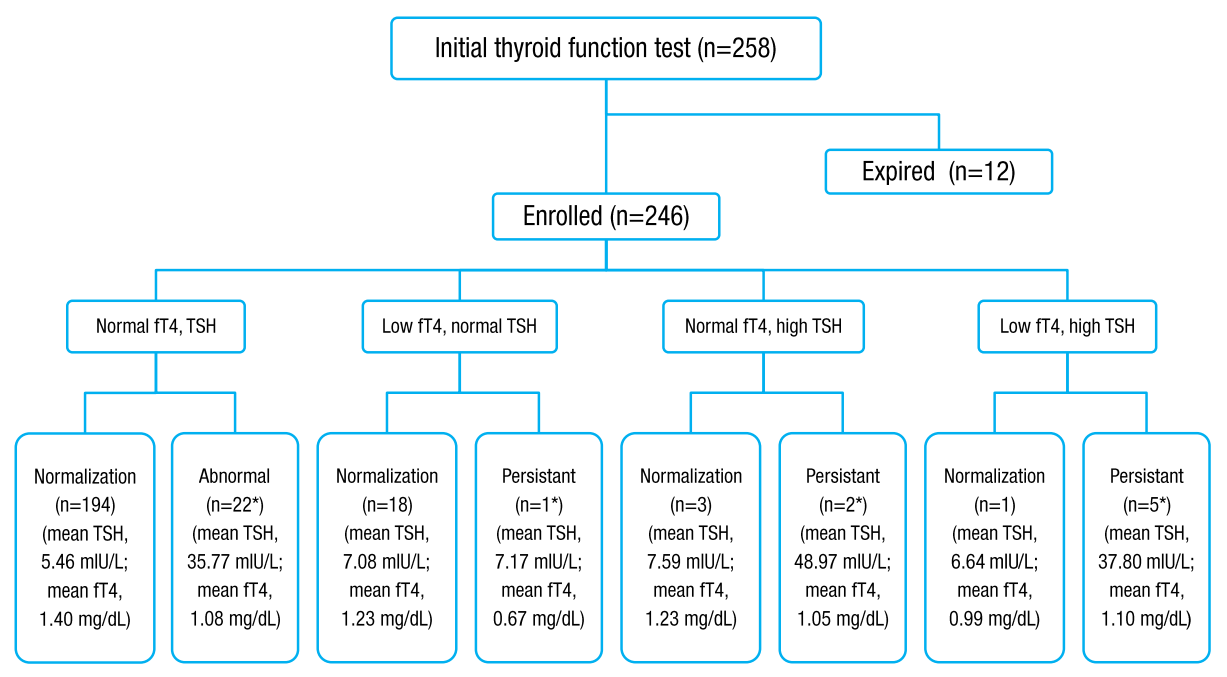

Fig. 1. Outcome of infants with thyroid function tests. A total of 246 prematurity were enrolled in this study and divided by 8 groups. Levothyroxine was prescribed to 30 patients (12.2\%). fT4, free thyroxine; TSH, thyroid-stimulating hormone. ${ }^{*}$ Requiring thyroid hormone replacement.

survived were enrolled and categorized into eight subgroups, according to results of thyroid function tests by the American Academy of Pediatrics, American Thyroid Association, and Lawson Wilkins Pediatric Endocrine Society ${ }^{5,6)}$ (Fig. 1). In total, $12.2 \%$ (30 of 246) of subjects had thyroid dysfunction that required thyroid hormone replacement. Thirty infants were assigned to the treatment group receiving thyroid replacement therapy and 216 to the nontreatment group. The level of TSH in initial TFT was significantly different between the treatment group and the nontreatment group $(19.3 \pm 25 \mathrm{mIU} / \mathrm{L}$ vs. $5.63 \pm 4.4$ $\mathrm{mIU} / \mathrm{L}, P<0.01)$, but the free T4 level was not significantly different $(1.16 \pm 0.53 \mathrm{mg} / \mathrm{dL}$ vs. $1.32 \pm 0.67 \mathrm{mg} / \mathrm{dL}, P=0.234)$. The values of follow-up TFT after treatment with levothyroxine were $5.82 \pm 5.24 \mathrm{mIU} / \mathrm{L}$ in TSH and $1.44 \pm 0.42 \mathrm{mg} / \mathrm{dL}$ in free T4 in the treatment group. In the treatment group, 73.3\% (22 of 30) had normal initial TFT, elevated TSH in second sample, and persistent hyperthyrotropinemia requiring thyroid hormone replacement therapy. Of 19 hypothyroxinemia cases, 18 were transient, and one was permanent and in need thyroid hormone replacement therapy. Of 30 in the treatment group, two had initial hyperthyrotropinemia and five had congenital hypothyroidism with a low initial free T4 and elevated TSH. Only two of 246 subjects showed abnormal thyroid function in the newborn screening test. One, allocated to the nontreatment group had transient hyperthyrotropinemia and elevated TSH was normalized without treatment. The other, assigned to the treatment group, had persistent hyperthyrotropinemia requiring thyroid hormone replacement.

\section{Demographic factors}

Gestational age, gender ratio and Apgar score at 1 and 5
Table 1. Demographic factors

\begin{tabular}{lccc}
\hline Demographic factor & $\begin{array}{c}\text { Treatment } \\
\text { group } \\
(\mathrm{n}=30)\end{array}$ & $\begin{array}{c}\text { Nontreatment } \\
\text { group } \\
(\mathrm{n}=216)\end{array}$ & $\begin{array}{c}P \\
\text { value }\end{array}$ \\
\hline Gestational age $\left(\mathrm{wk}^{+\mathrm{day}}\right)$ & $28^{+1} \pm 3^{+2}$ & $28^{+3} \pm 2^{+5}$ & 0.632 \\
Birth weight (g) & $935 \pm 313$ & $1,060 \pm 269$ & 0.021 \\
Male sex & $11(36.7)$ & $109(50.5)$ & 0.157 \\
Apgar score at 1 min & $4.7 \pm 1.3$ & $4.3 \pm 1.5$ & 0.163 \\
Apgar score at 5 min & $6.6 \pm 1.2$ & $6.8 \pm 1.2$ & 0.374 \\
Small for gestational age & $11(36.7)$ & $29(13.4)$ & 0.001 \\
Antenatal magnesium sulfate therapy & $15(50.0)$ & $110(50.9)$ & 0.924 \\
Antenatal corticosteroid therapy & $21(70.0)$ & $166(76.9)$ & 0.410 \\
Maternal PIH & $5(16.7)$ & $44(20.4)$ & 0.634 \\
Maternal GDM & $0(0)$ & $13(6)$ & 0.167 \\
\hline Values are presented as mean \pm standard deviation or number (\%). \\
PIH, pregnancy induced hypertension; GDM, gestational diabetes mellitus.
\end{tabular}

minutes were not different between the two groups. Antenatal magnesium sulfate therapy, antenatal corticosteroid therapy, maternal GDM, and maternal PIH were not significantly different between the two groups. Birth weight was lower in the treatment group compared to the nontreatment group $(935 \pm 313 \mathrm{~g}$ vs. $1060 \pm$ $269 \mathrm{~g}, P=0.021)$. SGA was more frequent in the treatment group than nontreatment group (36.7\% vs. $13.4 \%, P=0.001$ ). There were more extremely low birth weight infants (ELBWIs) in the treatment group, but the difference was not statistically significant (56.7\% vs. $38.4 \%, P=0.057$ ) (Table 1).

\section{Morbidities and clinical outcomes}

RDS, PDA, sepsis, IVH ( $\geq$ grade III), NEC ( $\geq$ stage II), ROP (requiring laser therapy), postnatal steroids therapy and BPD 
Table 2. Morbidity and outcomes

\begin{tabular}{lccc}
\hline Variable & $\begin{array}{c}\text { Treatment } \\
\text { group } \\
(\mathrm{n}=30)\end{array}$ & $\begin{array}{c}\text { Nontreatment } \\
\text { group } \\
(\mathrm{n}=216)\end{array}$ & $\begin{array}{c}P \\
\text { value }\end{array}$ \\
\hline Respiratory distress syndrome & $25(83.3)$ & $193(89.4)$ & 0.331 \\
Patent ductus artreriosus & $18(60)$ & $131(60.6)$ & 0.946 \\
Sepsis & $6(20)$ & $32(14.8)$ & 0.462 \\
IVH ( $\geq$ grade III) & $5(16.7)$ & $21(9.7)$ & 0.246 \\
NEC ( $\geq$ stage II) & $1(3.3)$ & $4(1.9)$ & 0.590 \\
ROP (requiring laser op.) & $9(30.0)$ & $39(18.1)$ & 0.122 \\
Postnatal steroid therapy & $10(33.3)$ & $70(32.4)$ & 0.919 \\
BPD ( $\geq$ moderate) & $6(20.0)$ & $20(9.3)$ & 0.073 \\
Full enteral feeding day (day) & $24 \pm 13$ & $21 \pm 11$ & 0.217 \\
Duration of PN (day) & $22 \pm 15$ & $19 \pm 15$ & 0.300 \\
Duration of mechanical ventilation (day) & $24 \pm 19$ & $20 \pm 12$ & 0.379 \\
Hospital stay (day) & $79 \pm 32$ & $69 \pm 25$ & 0.042 \\
\hline Values are presented as meantstandard & &
\end{tabular}

Values are presented as mean \pm standard deviation or number (\%).

IVH, intraventricular hemorrhage; NEC, necrotizing enterocolitis; ROP retinopathy of prematurity; BPD, bronchopulmonary dysplasia; PN, parentera nutrition.

(moderate to severe) were not significantly different between the two groups. Full enteral feeding day, duration of parenteral nutrition and duration of mechanical ventilation were not different. Prolonged hospital stay was observed in the treatment group (79 days vs. 69 days, $P=0.042$ ) (Table 2).

\section{Discussion}

The fetal hypothalamic-pituitary-thyroid axis begins to function after the first trimester and is completed at the term gestation. As a result, hypothyroidism is common in preterm infants due to immaturity of thyroid hormonal regulation and increased demand for thyroid hormone by certain diseases of preterm infants, not congenital abnormalities of the thyroid gland $d^{3)}$. Some cases of thyroid dysfunction in preterm infants are transient and resolved with postnatal maturation without thyroid hormone treatment ${ }^{13)}$.

Transient hypothyroxinemia is self-limiting and more common in preterm infants, occuring in 20\% of preterm infants with a gestational age $<34$ weeks and 29\% in VLBWIs with a gestational age $<32$ weeks ${ }^{14,15)}$. The benefits of thyroid hormone treatment for transient hypothyroxinemia in preterm infants remain unknown and the optimal follow-up duration has not been determined. Therefore, it is unclear whether the administration of thyroid hormone in preterm infants reduces neonatal morbidity and mortality or improves neurodevelopmental outcome. Studies reported that prophylactic replacement of thyroid hormone in preterm infants is ineffective and does not lower neonatal morbidity, mortality or neurodevelopmental disabilities ${ }^{16,177}$.
Transient hypothyroxinemia is more common in the smallest and least mature preterm infants with an increased risk of neurodevelopmental disability and cerebral palsy and decreased mental developmental index and psychomotor developmental index. Van Wassenaer and $\mathrm{Kok}^{3)}$ reported that low thyroid hormone levels may be associated with neurodevelopmental impairments in preterm infants. They previously reported that low free T4 levels in the first postnatal month were related to poor neurodevelopmental outcome in preterm infants with a gestational age $<30$ weeks $^{18}$.

It is unclear whether thyroid hormone treatment improves neurodevelopmental outcome or thyroid dysfunction in preterm infants. Delayed replacement of thyroid hormone in infants with congenital hypothyroidism may have a significant adverse effect on the neurodevelopmental outcome of high-risk preterm infants ${ }^{19)}$. The evolution of congenital hypothyroidism to transient or permanent is difficult to predict. Further studies are required to determine whether transient hypothyroxinemia of prematurity affects neurodevelopmental outcome, and the effectiveness and extent of thyroid hormone treatment in preterm infants.

Preterm infants in NICUs are subjected to concomitant drugs, such as dopamine, metoclopramide, aminophylline and steroids to treat their illness, which result in transient thyroid dysfunction and affect screening test results ${ }^{13,20}$. Metoclopramide, a dopamine receptor antagonist used to prompt gastric emptying in gastrointestinal dysmotility, induces a significant TSH release that results in thyroid dysfunction ${ }^{20}$. Aminophylline and caffeine, and their active metabolite theophylline, are used as respiratory stimulants in apnea of prematurity. They increase the expression of TSH and thyroglobulin that result in thyroid dysfunction ${ }^{211}$. Antenatal steroids reduce the risk of RDS and postnatal steroids to accelerate lung maturation. Steroids have various effects on thyroid function, such as inhibition of TSH release, reduction of free T4 and TBG, decreased conversion of T4 to T3 and increased renal iodine clearance. Steroids may contribute to poor neurodevelopmental outcome through adverse side effects or by inducing thyroid dysfunction ${ }^{22-24}$. This study found no difference in frequency between the nontreatment group and treatment group administered antenatal and postnatal corticosteroid.

Preterm infants have a higher risk of contracting certain diseases. The effect of RDS on serum thyroid hormone levels remains unresolved, and studies revealed conflicting findings. Some studies suggested that RDS may affect thyroid dysfunc$\operatorname{tion}^{25,26)}$, while others concluded that there is no or marginal association between RDS and thyroid dysfunction ${ }^{211}$. Williams et al. ${ }^{21)}$ reported that late onset sepsis markedly decreased T4, T3, and TBG. They say that RDS, IVH, NEC, as well as late onset sepsis affect thyroid hormone level and thyroid dysfunction induced by acute inflammatory cytokine response. Carrascosa et $\mathrm{al}^{2)}$ concluded that hypothyroidism is more common in sick 
preterm infants with PDA and treated with inotropics. In this study, the incidence of RDS, IVH ( $\geq$ grade III), NEC ( $\geq$ stage II), sepsis, and PDA were not significantly increased in the treatment group needing thyroid hormone replacement. Compared to the nontreatment group, ELBWIs were more frequent in the treatment group without statistical difference, and infants with SGA were more frequent in the treatment group in our study. Based on these results SGA or ELBWIs may have more thyroid dysfunction. Van Wassenaer and $\mathrm{Kok}^{3)}$ reported similar results, which found that smaller and less mature preterm infants have significantly lower T3 and free T4 levels than more mature preterm infants or term infants.

In one study, $0.4 \%$ (44 of 2,647) of VLWBIs with a gestational age $<32$ weeks had elevated TSH, 66\% (29 of 44) elevated TSH was delayed TSH elevation and 25\% (11 of 44) elevated TSH requiring thyroid hormone replacement therapy ${ }^{27)}$. Five of 11 requiring thyroid hormone therapy were found in the second specimen, and five in the third specimen. The researchers worried that neonatal screening for congenital hypothyroidism is not well established in preterm infants. They concluded that specific protocol regarding routine resample to rescreen congenital hypothyroidism in VLBWIs with a gestational age $<32$ weeks is required. In this study, 9.8\% (24 of 246) of VLBWIs had elevated TSH and 91.6\% (22 of 24) had delayed TSH elevation. The 22 VLBWIs with delayed TSH elevation had normal TFT in the first sample, and elevated TSH in the second sample. Had retesting not been performed, thyroid dysfunction in VLBWIs would have remained undetected.

Screening programs for congenital hypothyroidism vary by country, with each selecting form the TSH method, T4 method, or the TSH and T4 combination. The ideal screening method is combined TSH and T4, which is more costly than a primary TSH or T4 method. However, primary TSH test can fail to identify TBG deficiency, central hypothyroidism, hypothyroxinemia with delayed TSH elevation, which is more common in preterm infants with low birth weight or very low birth weight. Mandel et al. ${ }^{28)}$ suggested that T4 measurements should be performed routinely with second blood specimens from all low birth weight infants and VLBWIs after screening with a primary TSH method. A primary T4 test can fail to identify infants with normal T4 and increased TSH. A combined TSH and T4 test is the ideal screening approach, circumventing potential limitations of TSH or T4 measurements, when screening for congenital hypothyroidism.

The newborn screening test in Korea only includes TSH, not the free T4 level due to cost effectiveness. In some states of United States, TSH and T4 levels in VLBWIs have been measured at 2, 6, and 10 weeks postnatal in addition to initial newborn screening since 1996, as set by policies of the New England Newborn Screening Program ${ }^{28)}$. Larson et al. ${ }^{29)}$ reported that retesting thyroid function of VLBWIs in the NICU is important, because not all screening programs measure T4 or track low T4. Thyroid function of VLBWIs be retested at 2 and 4 weeks postnatal to maximize detection of congenital hypothyroidism and start medication early.

In conclusion, thyroid dysfunction in VLBWIs is common. In this study, more than 10\% of VLBWIs (12.2\%, 30 of 246) had thyroid dysfunction that required thyroid hormone replacement. However, only one patient registered positive for abnormal thyroid function in the newborn screening test with TSH. The cost of diagnosing congenital hypothyroidism at an older age, and treating and educating a developmentally impaired person at an older age due to untreated congenital hypothyroidism, can be much higher than the cost of screening with a combined TSH and T4 method or retesting thyroid function in VLBWIs ${ }^{5)}$. Therefore, we recommend retesting thyroid function with TSH and free T4 measurements in high risk preterm infants that presented normal initial TSH level in newborn screening test. Physicians should not rule out hypothyroidism if signs or symptoms suggest hypothyroidism, even when the newborn screening test reports normal thyroid function. Serum free T4 and TSH measurements with second or third specimens should be performed when clinical symptoms suggest hypothyroidism, despite newborn screening results.

\section{Conflict of interest}

No potential conflict of interest relevant to this article was reported.

\section{References}

1. Simoneau-Roy J, Marti S, Deal C, Huot C, Robaey P, Van Vliet G. Cognition and behavior at school entry in children with congenital hypothyroidism treated early with high-dose levothyroxine. J Pediatr 2004;144:747-52.

2. Carrascosa A, Ruiz-Cuevas P, Clemente M, Salcedo S, Almar J. Thyroid function in 76 sick preterm infants 30-36 weeks: results from a longitudinal study. J Pediatr Endocrinol Metab 2008;21: 237-43.

3. van Wassenaer AG, Kok JH. Hypothyroxinaemia and thyroid function after preterm birth. Semin Neonatol 2004;9:3-11.

4. Vigone MC, Caiulo S, Di Frenna M, Ghirardello S, Corbetta C, Mosca F, et al. Evolution of thyroid function in preterm infants detected by screening for congenital hypothyroidism. J Pediatr 2014;164:1296-302.

5. American Academy of Pediatrics, Rose SR; Section on Endocrinology and Committee on Genetics, American Thyroid Association, Brown RS; Public Health Committee, Lawson Wilkins Pediatric Endocrine Society, Foley T, et al. Update of newborn screening and therapy for congenital hypothyroidism. Pediatrics 2006;117:2290-303.

6. Guy VV, Johnny D. Disorders of the thyroid in the newborn and infant. In: Sperling MA, editor. Pediatric endocrinology. 4th ed. 
Philadelphia (PA): Saunders, 2014:186-208.

7. Lubchenco LO, Hansman C, Dressler M, Boyd E. Intrauterine growth as estimated from liveborn birth-weight data at 24 to 42 weeks of gestation. Pediatrics 1963;32:793-800.

8. Stoll BJ, Hansen NI, Adams-Chapman I, Fanaroff AA, Hintz SR, Vohr B, et al. Neurodevelopmental and growth impairment among extremely low-birth-weight infants with neonatal infection. JAMA 2004;292:2357-65.

9. Papile LA, Burstein J, Burstein R, Koffler H. Incidence and evolution of subependymal and intraventricular hemorrhage: a study of infants with birth weights less than 1,500 gm. J Pediatr 1978;92:529-34.

10. Walsh MC, Kliegman RM. Necrotizing enterocolitis: treatment based on staging criteria. Pediatr Clin North Am 1986;33:179-201.

11. An international classification of retinopathy of prematurity. Pediatrics 1984;74:127-33.

12. Kinsella JP, Greenough A, Abman SH. Bronchopulmonary dysplasia. Lancet 2006;367:1421-31.

13. Chung ML, Yoo HW, Kim KS, Lee BS, Pi SY, Lim G, et al. Thyroid dysfunctions of prematurity and their impacts on neurodevelopmental outcome. J Pediatr Endocrinol Metab 2013;26:449-55.

14. Delahunty C, Falconer S, Hume R, Jackson L, Midgley P, Mirfield $\mathrm{M}$, et al. Levels of neonatal thyroid hormone in preterm infants and neurodevelopmental outcome at 5 1/2 years: millennium cohort study. J Clin Endocrinol Metab 2010;95:4898-908.

15. Dilli D, Eras Z, Andiran N, Dilmen U, Sakrucu ED. Neurodevelopmental evaluation of very low birth weight infants with transient hypothyroxinemia at corrected age of 18-24 months. Indian Pediatr 2012;49:711-5.

16. Osborn DA, Hunt RW. Postnatal thyroid hormones for preterm infants with transient hypothyroxinaemia. Cochrane Database Syst Rev 2007;(1):CD005945.

17. Osborn DA, Hunt RW. Prophylactic postnatal thyroid hormones for prevention of morbidity and mortality in preterm infants. Cochrane Database Syst Rev 2007;(1):CD005948.

18. van Wassenaer AG, Briet JM, van Baar A, Smit BJ, Tamminga P, de Vijlder JJ, et al. Free thyroxine levels during the first weeks of life and neurodevelopmental outcome until the age of 5 years in very preterm infants. Pediatrics 2002;110:534-9.

19. Seth A. Transient hypothyroxinemia of prematurity does it have clinical relevance? Indian Pediatr 2012;49:703-4.

20. Lombardi G, Iodice M, Miletto P, Merola B, Panza N, Annunziato L. Prolactin and TSH response to TRH and metoclopramide before and after l-thyroxine therapy in subclinical hypothyroidism. Neuroendocrinology 1986;43:676-8.

21. Williams FL, Ogston SA, van Toor H, Visser TJ, Hume R. Serum thyroid hormones in preterm infants: associations with postnatal illnesses and drug usage. J Clin Endocrinol Metab 2005;90:595463.

22. Williams FL, Simpson J, Delahunty C, Ogston SA, BongersSchokking JJ, Murphy N, et al. Developmental trends in cord and postpartum serum thyroid hormones in preterm infants. J Clin Endocrinol Metab 2004;89:5314-20.

23. Yeh TF, Lin YJ, Lin HC, Huang CC, Hsieh WS, Lin CH, et al. Outcomes at school age after postnatal dexamethasone therapy for lung disease of prematurity. N Engl J Med 2004;350:1304-13.

24. Friedman S, Shinwell ES. Prenatal and postnatal steroid therapy and child neurodevelopment. Clin Perinatol 2004;31:529-44.

25. Cuestas RA, Engel RR. Thyroid function in preterm infants with respiratory distress syndrome. J Pediatr 1979;94:643-6.

26. Abbassi V, Merchant K, Abramson D. Postnatal triiodothyronine concentrations in healthy preterm infants and in infants with respiratory distress syndrome. Pediatr Res 1977;11:802-4.

27. Silva SA, Chagas AJ, Goulart EM, Silva GA, Marcal LV, Gomes $\mathrm{MN}$, et al. Screening for congenital hypothyroidism in extreme premature and/or very low birth weight newborns: the importance of a specific protocol. J Pediatr Endocrinol Metab 2010;23:45-52.

28. Mandel SJ, Hermos RJ, Larson CA, Prigozhin AB, Rojas DA, Mitchell ML. Atypical hypothyroidism and the very low birthweight infant. Thyroid 2000;10:693-5.

29. Larson C, Hermos R, Delaney A, Daley D, Mitchell M. Risk factors associated with delayed thyrotropin elevations in congenital hypothyroidism. J Pediatr 2003;143:587-91. 